PREPARED FOR THE U.S. DEPARTMENT OF ENERGY, UNDER CONTRACT DE-AC02-76CH03073

PPPL-3977

PPPL-3977

UC-70

Criteria for Second Stability for Ballooning Modes in Stellarators

by

S.R. Hudson and C.C. Hegna

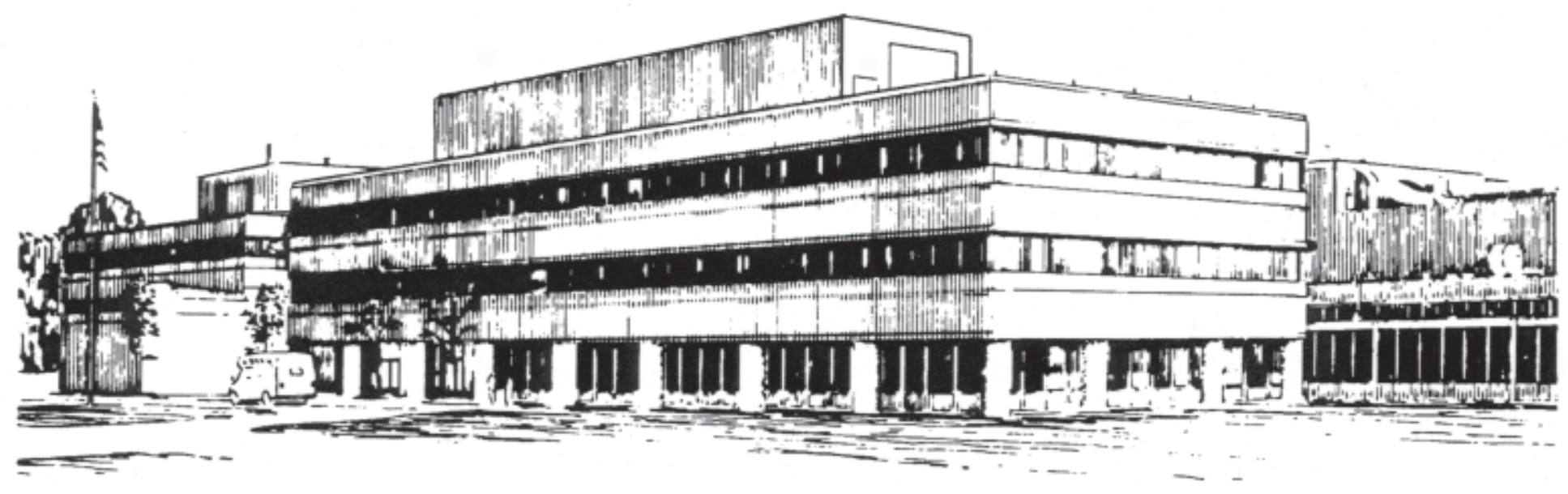

PRINCETON PLASMA PHYSICS LABORATORY PRINCETON UNIVERSITY, PRINCETON, NEW JERSEY 


\section{PPPL Reports Disclaimer}

This report was prepared as an account of work sponsored by an agency of the United States Government. Neither the United States Government nor any agency thereof, nor any of their employees, makes any warranty, express or implied, or assumes any legal liability or responsibility for the accuracy, completeness, or usefulness of any information, apparatus, product, or process disclosed, or represents that its use would not infringe privately owned rights. Reference herein to any specific commercial product, process, or service by trade name, trademark, manufacturer, or otherwise, does not necessarily constitute or imply its endorsement, recommendation, or favoring by the United States Government or any agency thereof. The views and opinions of authors expressed herein do not necessarily state or reflect those of the United States Government or any agency thereof.

\section{Availability}

This report is posted on the U.S. Department of Energy's Princeton Plasma Physics Laboratory Publications and Reports web site in Fiscal Year 2004. The home page for PPPL Reports and Publications is: http://www.pppl.gov/pub_report/

DOE and DOE Contractors can obtain copies of this report from:

U.S. Department of Energy

Office of Scientific and Technical Information

DOE Technical Information Services (DTIS)

P.O. Box 62

Oak Ridge, TN 37831

Telephone: (865) 576-8401

Fax: (865) 576-5728

Email: reports@adonis.osti.gov

This report is available to the general public from:

National Technical Information Service

U.S. Department of Commerce

5285 Port Royal Road

Springfield, VA 22161

Telephone: $1-800-553-6847$ or

(703) $605-6000$

Fax: (703) 321-8547

Internet: http://www.ntis.gov/ordering.htm 


\title{
Criteria for second stability for ballooning modes in stellarators
}

\author{
S.R.Hudson \& C.C.Hegna \\ Princeton Plasma Physics Laboratory, PO Box 451, Princeton NJ 08543. \\ Department of Engineering Physics, University of Wisconsin-Madison, WI 53706.
}

(Dated: June 11, 2004)

An expression determining how variations in the pressure-gradient and average magnetic shear affect ballooning stability for a stellarator equilibrium is presented. The procedure for determining the marginal stability boundaries, for each field line, depends only on the equilibrium and a single ballooning eigenfunction calculation. This information is sufficient to determine if increasing pressure-gradient is stabilizing or destabilizing and to predict whether the configuration possess a second stable region. 
An economically viable fusion reactor must sustain high-pressure, stable equilibria. It is often predicted that the short wavelength pressure-driven instability, the ballooning mode, will be the instability that limits the obtainable plasma stored energy. This letter will present an expression describing how ballooning stability will vary as an arbitrary threedimensional equilibrium is varied and predicts whether a configuration will possess a second stable region, in which equilibria may possess arbitrarily large pressure-gradients and not be limited by ballooning instability. The technique depends on a two-stage approach. Initially, the method of profile variations is used to construct families of neighboring magnetostatic equilibria $[1,2]$. Subsequently, a perturbation approach is employed to estimate the effect these variations have on the ballooning eigenvalue.

Second stability is the paradoxical phenomenon where increased pressure-gradient can stabilize ballooning modes. The effect of second stability has long been known in the (axisymmetric) tokamak community [1], but it is not clear how the (non-axisymmetric) stellarator will behave. The three dimensional geometry of stellarators gives rise to increased complexity in equilibrium and stability calculations. Indeed, the complexity requires equilibrium and stability studies to be performed numerically at significant computational cost, a fact which highlights the importance of this work: that analytic predictions of stability can be derived.

An equilibrium is obtained when the Lorentz force balances the pressure-gradient force, $\mathbf{J} \times \mathbf{B}=\nabla p$. The equilibrium is determined by the plasma boundary, the pressure profile, and an additional profile quantity such as the current density or rotational-transform. The rotational-transform, $t$, measures the pitch of the magnetic field lines as they twist around the torus. By considering a small displacement, of the form $\boldsymbol{\xi}(\mathbf{x}) \exp (-i \omega t)$, from an equilibrium, linear stability is determined by an eigenvalue equation, $-\omega^{2} \rho \boldsymbol{\xi}=\mathbf{F}(\boldsymbol{\xi})$, where $\mathbf{F}=\mathbf{J} \times \delta \mathbf{B}+\delta \mathbf{J} \times \mathbf{B}-\nabla \delta p$. If this equation allows a growth rate such that $\omega^{2}<0$, the perturbation will grow.

To study ballooning modes, the WKB eikonal representation $\boldsymbol{\xi}=\hat{\boldsymbol{\xi}} \exp [i n S]$ is employed $[3,4]$, where $n$ is the (large) toroidal mode number, $\mathbf{k}=\nabla S(\mathbf{x})$ is the wave vector, and to lowest order in $1 / n$ it is assumed that $\mathbf{k} \cdot \mathbf{B}=0$. The stability condition reduces to an eigenvalue problem, the ballooning equation, which is local to a field line and simpler to solve numerically. Of primary importance is the boundary between stable and unstable equilibria: the marginal stability boundary. 
In this letter we consider how the stability of an arbitrary equilibrium will vary, as the equilibrium itself is varied. In particular, an analytic expression for how the ballooning growth rate, of a selected field line with given radial wave vector, depends on small variations in the pressure-gradient, $\delta p^{\prime}$, and rotational-transform-gradient (the average magnetic shear), $\delta t^{\prime}$, is derived. This expression allows the marginal stability boundary for the selected field line in the given equilibrium to be determined immediately, and can also predict the existence, or otherwise, of the second stable region.

Recent work on the topic of second stability has indicated that some stellarator configurations do possess second stability [5], and that some do not [6]. The question thus arises : what property of the configuration determines whether a second stability region will exist?

The 'brute-force' approach is to numerically compute an equilibrium and solve the ballooning eigenvalue equation. The pressure is then increased and the process repeated. This process is tedious and imparts little insight. More importantly perhaps, is that this method cannot ascertain if whether, beyond a region of instability, there lies a second stable region.

A better approach, the method of profile variations, was introduced by Greene \& Chance [1] for axisymmetric configurations. They considered variations in the pressure-gradient and average shear at a selected magnetic surface in the equilibrium. The pressure-gradient and average shear have a crucial impact on ballooning stability, as the presence of pressuregradients in regions of unfavorable curvature is the cause of ballooning instability, while shear is the dominant stabilizing mechanism. The equilibrium itself is then adjusted to preserve force balance, and a family of semi-analytic neighboring equilibria is constructed. For each such constructed equilibrium, the ballooning equation may be re-solved numerically (exactly) and marginal stability diagrams constructed. Such diagrams are widely used to study tokamak stability, and recently the analysis has been extended to stellarator geometry $[2,7]$. This method eliminates the need to re-compute the equilibrium, and illuminates the role of the local magnetic shear.

The mechanism for second stability was determined to be that pressure induced variations in the parallel current, $J_{\|}=\mathbf{J} \cdot \mathbf{B} / B^{2}$, cause variations in the local shear, which may strengthen the stabilizing force in regions of unfavorable curvature. A related pressureinduced stabilization phenomenon that should be mentioned, is when increased pressure alters the geometry of the configuration [8-10]. While this mechanism can modify the stability properties, it is generally a smaller effect, as is verified by brute force equilibrium 
reconstruction and stability analysis [11].

In this letter we build upon the method of profile variations and make the realization that it is not necessary to re-solve the ballooning equation for the semi-analytically constructed equilibria. Whether ballooning stability will improve or degrade as the pressure-gradient is increased can be inferred from information obtained directly from the original equilibrium. An expression for how the ballooning eigenvalue depends on variations in the pressuregradient and average shear is derived.

Following Hegna \& Nakajima [2], hereafter HN, we consider an equilibrium expressed in Boozer coordinates $(\psi, \theta, \zeta)$, being the toroidal flux function, the poloidal angle and the toroidal angle respectively [12]. In these coordinates, the magnetic field takes the form

$$
\mathbf{B}=\nabla \psi \times \nabla(\theta-t \zeta)=G \nabla \zeta+I \nabla \theta+h \nabla \psi
$$

where $t(\psi)$ is the rotational transform, $G(\psi)$ and $I(\psi)$ are the poloidal current outside and the toroidal current inside the surface $\psi$, and the function $h(\psi, \theta, \zeta)$ is related to the pressure-induced parallel-currents, the Pfirsch-Schlüter currents. Given this representation of the magnetic field, it is the coordinate transformation $\mathbf{x}(\psi, \theta, \zeta)$ which implicitly defines the equilibrium.

Employing the angle variables $\alpha=\theta-t \zeta$ and $\eta=\zeta$, where $\alpha$ labels the field line and $\eta$ labels position along the field line, the leading order solution for the fluid displacement $\xi$ is governed by an ordinary differential equation along the field line [4]

$$
\partial_{\eta} P \partial_{\eta} \xi+Q \xi=\gamma R \xi
$$

where $\gamma=-\omega^{2}$ is the local eigenvalue and the ballooning coefficients are given by [2]

$$
\begin{aligned}
& P=\frac{B^{2}}{g^{\psi \psi}}+g^{\psi \psi} L^{2}, \\
& Q=2 p^{\prime} \sqrt{g}\left(G+{ }_{t} I\right)\left(\kappa_{n}+\kappa_{g} L\right),
\end{aligned}
$$

where $R=\sqrt{g}^{2} P, p^{\prime}=d p / d \psi$ is the pressure-gradient, $g^{\psi \psi}=\nabla \psi \cdot \nabla \psi, \sqrt{g}$ is the Jacobian, $\kappa_{n}, \kappa_{g}$ are the normal and geodesic curvatures and $L$ is the integrated local shear

$$
L=\int_{\eta_{k}}^{\eta} d \eta^{\prime} s\left(\eta^{\prime}\right)
$$

The local shear is written

$$
s=t^{\prime}+\frac{\partial}{\partial \eta}\left(\frac{G g_{\psi \theta}-I g_{\psi \zeta}}{\sqrt{g} g^{\psi \psi}}\right)
$$


where $g_{\psi \theta}=\mathbf{e}_{\psi} \cdot \mathbf{e}_{\theta}$ and $g_{\psi \zeta}=\mathbf{e}_{\psi} \cdot \mathbf{e}_{\zeta}$. This is an Sturm-Liouville eigenvalue equation with boundary condition $\xi( \pm \infty)=0$. To determine stability, it is necessary to determine the largest eigenvalue $\gamma$. In the following, we will solve the ballooning equation for a prescribed equilibrium and determine the unstable eigenvalue and its eigenfunction. Subsequently, this eigenfunction will be used to determine the stability properties as a function of variations in the plasma profiles. It should be emphasized in this procedure that only this one ballooning eigenvalue equation need be solved.

To study the effect of increasing pressure-gradient we follow HN, who applied the method of profile variations to stellarators, and introduce variations in the pressure-profile $p(\psi)$ and rotational-transform profile $t(\psi)$, at a selected surface $\psi=\psi_{b}$, of the form

$$
\begin{aligned}
& \bar{p}(\psi)=p(\psi)+\mu \delta p(y), \\
& \bar{t}(\psi)=t(\psi)+\mu \delta t(y),
\end{aligned}
$$

where $\mu$ is a small expansion parameter and barred quantities include the effect of the variations. The auxiliary variable $y=\left(\psi-\psi_{b}\right) / \mu$ is used to ensure that the variations in the pressure-gradient and average shear are $\mathcal{O}(1)$, whereas the variation in the pressure and rotational-transform are $\mathcal{O}(\mu)$. The rationale for imposing such variations is that it is the pressure-gradient and shear, rather than the pressure and rotational-transform, that directly influences ballooning stability. In the following, the notation $\delta p^{\prime}=d \delta p / d y, \delta t^{\prime}=d \delta t / d y$ (but $p^{\prime}=d p / d \psi, t^{\prime}=d t / d \psi$ ) is used.

All physically relevant quantities are similarly varied. The variations are constrained by requiring that the system satisfy $\nabla p=\mathbf{J} \times \mathbf{B}$ and that the magnetic field strength be undisturbed to lowest order. The coordinate transformation is written $\overline{\mathbf{x}}=\mathbf{x}(\psi, \theta, \zeta)+$ $\mu \delta \mathbf{x}(y, \theta, \zeta)$, with basis vectors

$$
\begin{array}{r}
\overline{\mathbf{e}}_{\psi}=\mathbf{e}_{\psi}+\partial_{y} \delta \mathbf{x}, \\
\overline{\mathbf{e}}_{\theta}=\mathbf{e}_{\theta}+\mu \partial_{\theta} \delta \mathbf{x}, \\
\overline{\mathbf{e}}_{\zeta}=\mathbf{e}_{\zeta}+\mu \partial_{\zeta} \delta \mathbf{x} .
\end{array}
$$

For consistency, both $G$ and $I$ are varied similarly to $p$ and $t$, and $h$ requires order unity variations. The $\mathcal{O}(1)$ quantity in the basis vector variations is $\partial_{y} \delta \mathbf{x}$, which is expanded in a basis defined by the magnetic field

$$
\frac{\partial \delta \mathbf{x}}{\partial y}=C \mathbf{B}+D \frac{\mathbf{B} \times \boldsymbol{\nabla} \psi}{B^{2}}+M \frac{\boldsymbol{\nabla} \psi}{g^{\psi \psi}} .
$$


Expressions for $C, D$ and $M$ are provided by HN. The term $D$ is particularly relevant and it is determined by the equation $\partial_{\eta} D=\delta t^{\prime} \partial_{\eta} D_{t^{\prime}}-\delta p^{\prime} \partial_{\eta} D_{p^{\prime}}$ with

$$
\begin{aligned}
\partial_{\eta} D_{t^{\prime}} & =\frac{1}{\oint 1 / g^{\psi \psi}}\left(\frac{1}{g^{\psi \psi}}-\oint \frac{1}{g^{\psi \psi}}\right) \\
\partial_{\eta} D_{p^{\prime}} & =\frac{V^{\prime}(G+t I)}{\oint 1 / g^{\psi \psi}}\left(\frac{\lambda}{g^{\psi \psi}} \oint \frac{1}{g^{\psi \psi}}-\frac{1}{g^{\psi \psi}} \oint \frac{\lambda}{g^{\psi \psi}}\right),
\end{aligned}
$$

where $4 \pi^{2} \oint Q \equiv \oint d \theta \oint d \zeta Q$ is the flux surface average, $\lambda$ is the ratio of the Pfirsch-Schlüter current to the pressure-gradient $\lambda=-\left(J_{\|}-\oint J_{\|}\right) / p^{\prime} V^{\prime}$, and $V^{\prime}$ is the average Jacobian. From Eqn.(6), it can be shown that the local shear has $\mathcal{O}(1)$ variations and takes the form $\bar{s}=s+\delta t^{\prime}+\delta t^{\prime} \partial_{\eta} D_{t^{\prime}}-\delta p^{\prime} \partial_{\eta} D_{p^{\prime}}$, and the integrated local shear is written

$$
\bar{L}=L+\delta t^{\prime} L_{t^{\prime}}+\delta p^{\prime} L_{p^{\prime}}
$$

where $L_{t^{\prime}}=\eta+D_{t^{\prime}}$ and $L_{p^{\prime}}=-D_{p^{\prime}}$.

As far as the ballooning equation is concerned, it is only the local shear, and of course $p^{\prime}$ and $t^{\prime}$, that is altered by the variations. In particular, the normal and geodesic curvatures $\kappa_{n}=[(\mathbf{b} \cdot \nabla) \mathbf{b} \cdot \nabla \psi] / g^{\psi \psi}$ and $\kappa_{g}=[(\mathbf{b} \cdot \nabla) \mathbf{b} \cdot \mathbf{B} \times \nabla \psi] / B^{2}$, as defined by HN, are unchanged to lowest order, since from Eqns(9-11) the variations in the unit vector in the direction of the magnetic field $\mathbf{b}=\left(t \mathbf{e}_{\theta}+\mathbf{e}_{\zeta}\right) /\left|t \mathbf{e}_{\theta}+\mathbf{e}_{\zeta}\right|$ and $\nabla \psi=\left(\mathbf{e}_{\theta} \times \mathbf{e}_{\zeta}\right) / \sqrt{g}$ are $\mathcal{O}(\mu)$.

Due to the localized nature of the ballooning equation, it is sufficient to restrict attention to the single surface $\psi=\psi_{b}$. As such, Eqn.(15) is exact. Also, the analytic variations Eqn. $(7,8)$ are of such a form that $\delta p^{\prime}$ and $\delta t^{\prime}$ are zero order, $\mathcal{O}(1)$, in $\mu$. It follows that the $\delta p^{\prime 2}, \delta p^{\prime} \delta t^{\prime}$ and $\delta t^{\prime 2}$ terms appearing in $\bar{L}^{2}$ and elsewhere are also $\mathcal{O}(1)$, and it is consistent to keep all these terms. In fact is it necessary to keep the $\delta p^{2}, \delta p^{\prime} \delta t^{\prime}$ and $\delta t^{\prime 2}$ terms to capture the essence of the second stable region.

All the information required to solve the ballooning equation for the perturbed system is now known; however, rather than solve for the eigenvalue numerically, we can make further progress analytically. The profile variations alter the coefficients of the eigenvalue equation, and eigenvalue perturbation theory is applicable. Note that the following perturbation analysis is distinct from the Greene \& Chance construction of neighboring equilibria and does not depend on the formal expansion parameter $\mu$. For small variations $\delta p, \delta$, the 
perturbed eigenvalue is written

$$
\begin{aligned}
\delta \gamma & =\frac{\partial \gamma}{\partial p^{\prime}} \delta p^{\prime}+\frac{\partial \gamma}{\partial t^{\prime}} \delta t^{\prime} \\
& +\frac{\partial^{2} \gamma}{\partial p^{\prime 2}} \delta p^{\prime 2}+\frac{\partial^{2} \gamma}{\partial p^{\prime} \partial t^{\prime}} \delta p^{\prime} \delta t^{\prime}+\frac{\partial^{2} \gamma}{\partial t^{\prime 2}} \delta t^{\prime 2} .
\end{aligned}
$$

Using the shorthand notation, $\left\langle\xi_{1}|F| \xi_{2}\right\rangle \int \xi R \xi d \eta=\int \xi_{1} F \xi_{2} d \eta$, the first order derivatives are given by

$$
\begin{aligned}
& \frac{\partial \gamma}{\partial p^{\prime}}=\left\langle\xi\left|\partial_{\eta} \delta P_{p^{\prime}} \partial_{\eta}+\delta Q_{p^{\prime}}-\gamma \delta R_{p^{\prime}}\right| \xi\right\rangle \\
& \frac{\partial \gamma}{\partial t^{\prime}}=\left\langle\xi\left|\partial_{\eta} \delta Q_{t^{\prime}} \partial_{\eta}+\delta Q_{t^{\prime}}-\gamma \delta R_{t^{\prime}}\right| \xi\right\rangle
\end{aligned}
$$

To calculate the second order derivatives, it is required to determine the first order variations, $\delta \xi_{p^{\prime}}$ and $\delta \xi_{t^{\prime}}$, in the eigenfunction, which are solved from

$$
\begin{gathered}
B \delta \xi_{p^{\prime}}=\frac{\partial \gamma}{\partial p^{\prime}} R \xi-\left[\partial_{\eta} \delta P_{p^{\prime}} \partial_{\eta}+\delta Q_{p^{\prime}}-\gamma \delta R_{p^{\prime}}\right] \xi \\
B \delta \xi_{t^{\prime}}=\frac{\partial \gamma}{\partial t^{\prime}} R \xi-\left[\partial_{\eta} \delta P_{t^{\prime}} \partial_{\eta}+\delta Q_{t^{\prime}}-\gamma \delta R_{t^{\prime}}\right] \xi
\end{gathered}
$$

where $B=\left[\partial_{\eta} P \partial_{\eta}+Q-\gamma R\right]$. The second order derivatives are then given by

$$
\begin{aligned}
\frac{\partial^{2} \gamma}{\partial p^{\prime 2}} & =\left\langle\xi\left|\partial_{\eta} \delta P_{p^{\prime 2}} \partial_{\eta}+\delta Q_{p^{\prime 2}}-\gamma \delta R_{p^{\prime 2}}\right| \xi\right\rangle \\
& +\left\langle\xi\left|\partial_{\eta} \delta P_{p^{\prime}} \partial_{\eta}+\delta Q_{p^{\prime}}-\gamma \delta R_{p^{\prime}}\right| \delta \xi_{p^{\prime}}\right\rangle \\
& -\frac{\partial \gamma}{\partial p^{\prime}}\left(\left\langle\xi|R| \delta \xi_{p^{\prime}}\right\rangle+\left\langle\xi\left|\delta R_{p^{\prime}}\right| \xi\right\rangle\right), \\
\frac{\partial^{2} \gamma}{\partial p^{\prime} \partial t^{\prime}} & =\left\langle\xi\left|\partial_{\eta} \delta P_{p^{\prime} t^{\prime}} \partial_{\eta}+\delta Q_{p^{\prime} t^{\prime}}-\gamma \delta R_{p^{\prime} t^{\prime}}\right| \xi\right\rangle \\
& +\left\langle\xi\left|\partial_{\eta} \delta P_{p^{\prime}} \partial_{\eta}+\delta Q_{p^{\prime}}-\gamma \delta R_{p^{\prime}}\right| \delta \xi_{t^{\prime}}\right\rangle \\
& +\left\langle\xi\left|\partial_{\eta} \delta P_{t^{\prime}} \partial_{\eta}+\delta Q_{t^{\prime}}-\gamma \delta R_{t^{\prime}}\right| \delta \xi_{p^{\prime}}\right\rangle \\
& -\frac{\partial \gamma}{\partial p^{\prime}}\left(\left\langle\xi|R| \delta \xi_{t^{\prime}}\right\rangle+\left\langle\xi\left|\delta R_{t^{\prime}}\right| \xi\right\rangle\right) \\
& -\frac{\partial \gamma}{\partial t^{\prime}}\left(\left\langle\xi|R| \delta \xi_{p^{\prime}}\right\rangle+\left\langle\xi\left|\delta R_{p^{\prime}}\right| \xi\right\rangle\right), \\
\frac{\partial^{2} \gamma}{\partial t^{\prime 2}} & =\left\langle\xi\left|\partial_{\eta} \delta P_{t^{\prime 2}} \partial_{\eta}-\gamma \delta R_{t^{\prime 2}}\right| \xi\right\rangle \\
& +\left\langle\xi\left|\partial_{\eta} \delta P_{t^{\prime}} \partial_{\eta}+\delta Q_{t^{\prime}}-\gamma \delta R_{t^{\prime}}\right| \delta \xi_{t^{\prime}}\right\rangle \\
& -\frac{\partial \gamma}{\partial t^{\prime}}\left(\left\langle\xi|R| \delta \xi_{t^{\prime}}\right\rangle+\left\langle\xi\left|\delta R_{t^{\prime}}\right| \xi\right\rangle\right) .
\end{aligned}
$$

In the above equations, $\delta P_{p^{\prime}}=2 L L_{p^{\prime}}, \delta P_{t^{\prime}}=2 L L_{t^{\prime}}, \delta P_{p^{\prime 2}}=L_{p^{\prime}}^{2}, \delta P_{p^{\prime} t^{\prime}}=2 L_{p^{\prime}} L_{t^{\prime}}, \delta P_{t^{\prime 2}}=$ $L_{t^{\prime}}^{2}, \delta Q_{p^{\prime}}=2 \sqrt{g}\left(G+{ }_{t} I\right)\left(\kappa_{n}+\kappa_{g} L\right)+2 p^{\prime} \sqrt{g}\left(G+{ }_{t} I\right) \kappa_{g} L_{p^{\prime}}, \delta Q_{t^{\prime}}=2 p^{\prime} \sqrt{g}\left(G+{ }_{t} I\right) \kappa_{g} L_{t^{\prime}}$, 
$\delta Q_{p^{\prime 2}}=2 \sqrt{g}\left(G+{ }_{t} I\right) \kappa_{g} L_{p^{\prime}}$, and $\delta Q_{p^{\prime} \boldsymbol{t}^{\prime}}=2 \sqrt{g}\left(G+{ }_{t} I\right) \kappa_{g} L_{t^{\prime}}$. Throughout we use $R=\sqrt{g}^{2} P$, $R_{p^{\prime}}=\sqrt{g}^{2} P_{p^{\prime}}, R_{t^{\prime}}=\sqrt{g}^{2} P_{t^{\prime}}, \ldots$

These derivatives depend only on the initial equilibrium and the unperturbed eigenvalueeigenfunction pair. Once they have been calculated, the influence of pressure-gradient and average shear variations on ballooning stability is known, and the marginal stability boundary, defined by $\gamma+\delta \gamma=0$, may immediately be determined from Eqn.(16). Furthermore, noting that positive $\gamma$ indicates instability, and that increasing pressure-gradient corresponds to $\delta p^{\prime}<0$, the following criteria are obtained:

criterion (1) For a small increase $-\delta p^{\prime}$, the eigenvalue $\gamma$ will increase if $\partial \gamma / \partial p^{\prime}<0$ and decrease if $\partial \gamma / \partial p^{\prime}>0$.

criterion (2) A second stable region is indicated if $\partial^{2} \gamma / \partial p^{2}<0$.

To consider a realistic stellarator equilibrium, we use the VMEC [13] code to compute an equilibrium. To solve the ballooning equation, we adopt a finite difference method, as described by Sanchez et al. [14]. The eigenfunction is represented by a discrete set of $(2 N+1)$ points $\xi_{i}$ equally spaced along a selected field line on the 'full-grid' according to $\eta_{i}=-\eta_{\infty}+(i-1) \Delta$, with the grid-spacing $\Delta=\eta_{\infty} / N$ chosen to give about 100 grid points along the field line per poloidal transit, with the boundary conditions $\xi_{1}=\xi_{2 N+1}=0$, and where $\eta_{\infty}$ is chosen sufficiently large to contain the mode (several poloidal transits). The equation to be solved becomes a set of $2 N-1$ linear equations of the form

$$
\frac{P_{i+\frac{1}{2}}}{\Delta} \frac{\left(\xi_{i+1}-\xi_{i}\right)}{\Delta}-\frac{P_{i-\frac{1}{2}}}{\Delta} \frac{\left(\xi_{i}-\xi_{i-1}\right)}{\Delta}+Q_{i} \xi_{i}=\gamma R_{i} \xi_{i} .
$$

Here, $Q_{i}$ and $R_{i}$ are calculated on the full-grid at $\eta_{i}$, whereas $P_{i+\frac{1}{2}}$ is calculated on the half-grid $\eta_{i}+\Delta / 2$. This is a matrix equation, $M \xi=\gamma \xi$, where $M$ is tri-diagonal. The largest eigenvalue and its eigenfunction are then solved using standard numerical routines [14]. The same finite difference approximation is suitable for calculating what amounts to be inner products appearing in Eqns.(17-23) and the matrix inversion in Eqns.(19,20).

Shown in Fig.(1) is the stability diagram for a three field period, quasi-poloidal stellaratortokamak hybrid studied by Ware et al. [5]. The ratio of plasma pressure to magnetic pressure is $\beta=2.41 \%$. The VMEC representation of the equilibrium contains the harmonics $n=0,7$ for $m=0$ and $n=-7,7$ for $m=1,12$, where 100 radial surfaces have been used, and the Boozer coordinate representation contains the harmonics $n=0,56$ for $m=0$ and $n=-56,56$ for $m=1,103$. For this diagram, the symmetric field line $\alpha=0$ on the 
$\psi=0.1$ surface has been selected, with the 'ballooning-angle' $\eta_{k}=0$. The marginal stability curve obtained by re-solving the perturbed eigenvalue equation exactly (that is numerically) at $200 \times 200$ points on the $\left(\delta t^{\prime}, \delta p^{\prime}\right)$ space is compared to the stability curve obtained from Eqn.(16), which required a single eigenvalue equation to be solved. The quantitative agreement between the semi-analytic expression Eqn.(16) and the numerical value is very good, particularly considering the large variation in $\left(\delta t^{\prime}, \delta p^{\prime}\right) \sim\left(t^{\prime}, p^{\prime}\right)$. The small difference in these curves is due to higher order corrections, $\mathcal{O}\left(\delta p^{\prime 3}\right), \ldots, \mathcal{O}\left(\delta t^{\prime 3}\right)$, to the eigenvalue estimate.

The eigenvalue perturbation theory is valid for discrete (non-degenerate) eigenvalues and as such the theory is valid only for the unstable spectrum (though discretization will eliminate the continuous spectrum). This problem may be avoided by adjusting the pressuregradient using the method of profile variations to find an unstable eigenmode. The stability diagram may then be based on this point.

The analysis is completely general and applicable to axi-symmetric devices such as tokamaks, where it is known that shaped configurations possess stronger second-stable regions. The method has been applied to a variety of stellarators with similarly satisfactory results to that presented here. The analysis presented in this letter may be of great benefit to stellarator optimization routines and future stellarator designs, existing stellarator experiments, and also to the study of micro-instabilities which employs a similar eikonal approach.

We thank Andrew Ware, Steve Hirshman, Raul Sanchez and Neil Pomphrey for assistance. This work was supported in part by U.S. Department of Energy Contract No. DE-AC02-76CH03073 and grant No. DE-FG02-99ER54546. 
[1] J.M. Greene and M.S. Chance. Nucl. Fus., 21(4):453, 1981.

[2] C.C. Hegna and N. Nakajima. Phys. Plasmas, 5(5):1336, 1998.

[3] J.W.Connor, R.J.Hastie and J.B.Taylor. Phys. Rev. Lett., 40(6):396, 1978.

[4] R.L. Dewar and A.H. Glasser. Phys. Fluids, 26(10):3038, 1983.

[5] A.S. Ware, S.P. Hirshman, D.A. Spong, et al. Phys. Rev. Lett., 89(12):125003-1, 2002.

[6] C.C. Hegna and S.R. Hudson. Phys. Rev. Lett., 87(3):035001-1, 2001.

[7] S.R. Hudson and C.C. Hegna. Phys. Plasmas, 10(12):4716, 2003.

[8] J.H.Harris, M.Murakami, B.A.Carreras, et al. Phys. Rev. Lett., 63(12):1249, 1989.

[9] B.A. Carreras, N. Dominguez, V.E. Lynch, et al. Fusion Technology, 23:71, 1993.

[10] T. Matsumoto, Y. Nakamura, and M. Wakatani. Journal of the Physical Society of Japan, 62(11):3904, 1993.

[11] S.R. Hudson, C.C. Hegna, R. Torasso, and A. Ware. Plasma Physics and Controlled Fusion, $46: 869,2004$.

[12] A.H. Boozer. Phys. Fluids, 24(11):1999, 1981.

[13] S.P. Hirshman and O. Betancourt. J.Comp. Phys., 96(1):99, 1991.

[14] R. Sanchez, S.P. Hirshman, J.C. Whitson, and A.S. Ware. J.Comp. Phys., 161:576, 2000. 
CAPTION FIGURE 1 : Comparison of stability boundary obtained from the exact eigenvalue solution (solid) with that obtained from Eqn.(16) (dotted). The location of the original (unstable) equilibrium surface is indicated with a + . 


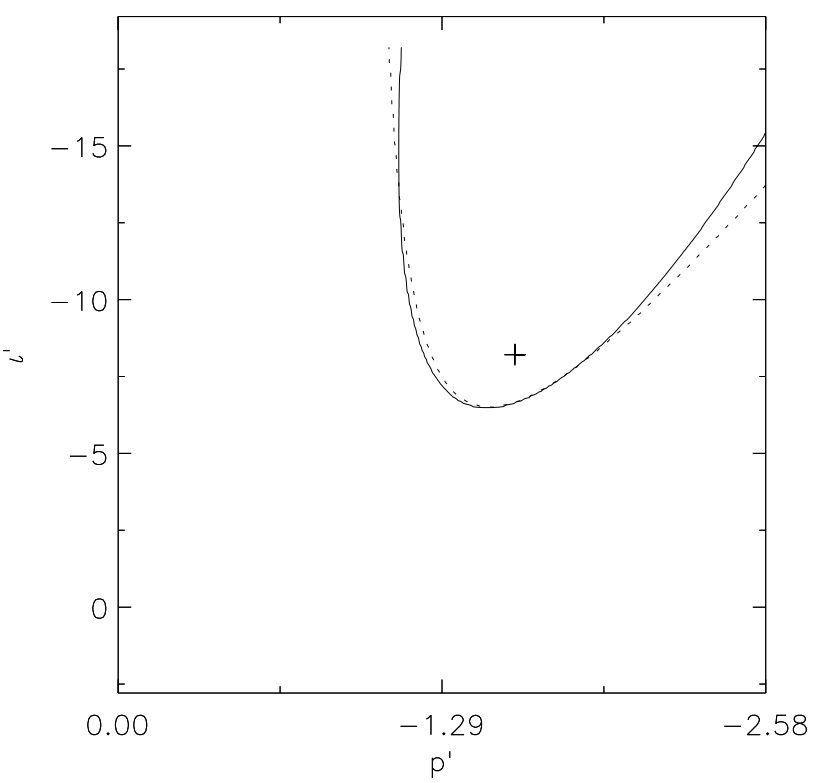

FIG. 1: 


\section{External Distribution}

Plasma Research Laboratory, Australian National University, Australia

Professor I.R. Jones, Flinders University, Australia

Professor João Canalle, Instituto de Fisica DEQ/IF - UERJ, Brazil

Mr. Gerson O. Ludwig, Instituto Nacional de Pesquisas, Brazil

Dr. P.H. Sakanaka, Instituto Fisica, Brazil

The Librarian, Culham Laboratory, England

Mrs. S.A. Hutchinson, JET Library, England

Professor M.N. Bussac, Ecole Polytechnique, France

Librarian, Max-Planck-Institut für Plasmaphysik, Germany

Jolan Moldvai, Reports Library, Hungarian Academy of Sciences, Central Research Institute for Physics, Hungary

Dr. P. Kaw, Institute for Plasma Research, India

Ms. P.J. Pathak, Librarian, Institute for Plasma Research, India

Ms. Clelia De Palo, Associazione EURATOM-ENEA, Italy

Dr. G. Grosso, Instituto di Fisica del Plasma, Italy

Librarian, Naka Fusion Research Establishment, JAERI, Japan

Library, Laboratory for Complex Energy Processes, Institute for Advanced Study, Kyoto University, Japan

Research Information Center, National Institute for Fusion Science, Japan

Dr. O. Mitarai, Kyushu Tokai University, Japan

Dr. Jiangang Li, Institute of Plasma Physics, Chinese Academy of Sciences, People's Republic of China

Professor Yuping Huo, School of Physical Science and Technology, People's Republic of China

Library, Academia Sinica, Institute of Plasma Physics, People's Republic of China

Librarian, Institute of Physics, Chinese Academy of Sciences, People's Republic of China

Dr. S. Mirnov, TRINITI, Troitsk, Russian Federation, Russia

Dr. V.S. Strelkov, Kurchatov Institute, Russian Federation, Russia

Professor Peter Lukac, Katedra Fyziky Plazmy MFF UK, Mlynska dolina F-2, Komenskeho Univerzita, SK-842 15 Bratislava, Slovakia

Dr. G.S. Lee, Korea Basic Science Institute, South Korea

Institute for Plasma Research, University of Maryland, USA

Librarian, Fusion Energy Division, Oak Ridge National Laboratory, USA

Librarian, Institute of Fusion Studies, University of Texas, USA

Librarian, Magnetic Fusion Program, Lawrence Livermore National Laboratory, USA

Library, General Atomics, USA

Plasma Physics Group, Fusion Energy Research Program, University of California at San Diego, USA

Plasma Physics Library, Columbia University, USA

Alkesh Punjabi, Center for Fusion Research and Training, Hampton University, USA

Dr. W.M. Stacey, Fusion Research Center, Georgia Institute of Technology, USA

Dr. John Willis, U.S. Department of Energy, Office of Fusion Energy Sciences, USA

Mr. Paul H. Wright, Indianapolis, Indiana, USA 
The Princeton Plasma Physics Laboratory is operated by Princeton University under contract with the U.S. Department of Energy.

\author{
Information Services \\ Princeton Plasma Physics Laboratory \\ P.O. Box 451 \\ Princeton, NJ 08543
}

Phone: 609-243-2750

Fax: 609-243-2751

e-mail: pppl_info@pppl.gov

Internet Address: http://www.pppl.gov 